\title{
Bone marrow aluminium storage in renal failure
}

\author{
MICHAEL KAYE \\ From the Division of Nephrology, The Montreal General Hospital, 1650 Cedar Avenue, Montreal, Quebec \\ H3G 1A4, Canada
}

SUMMARY Using the staining method for aluminium with the ammonium salt of aurine $\overrightarrow{\vec{\omega}}$ tricarboxylic acid, aluminon, 18 patients with end stage renal disease gave positive reactions in $\stackrel{\omega}{S}$ iliac crest bone biopsies and 11 of these had positive staining in the bone marrow. In one the 용 marrow was positive and the bone negative. The marrow reaction is putatively regarded as caused $\omega_{\infty}$ by aluminium storage in unidentified cells, possibly of the macrophage system which are strongly fluorescent when examined after prior tetracycline labelling. Marrow storage should be consi-: dered when assessing the bone aluminium burden.

The application by Buchanan et $a l^{1}$ of a specific staining technique for aluminium to bone sections from patients with renal failure has led to widespread use of this method for detection of aluminium storage in bone. ${ }^{23}$ None of these authors has noted the deposition of aluminium except in the bone itself, usually at the osteoid and mineral interface or less frequently along cement lines. While staining biopsy material for aluminium from patients with end stage renal disease we were surprised to find evidence of aluminium positive material present in the bone marrow and quite separate from the trabecular surfaces. This paper describes these findings.

\section{Material and methods}

Twenty-eight patients were studied for diagnostic purposes: nine had severe chronic renal failure and the remainder were on long term haemodialysis. Most patients were labelled with tetracycline prior to biopsy using a 2 days tetracycline -12 days off -4 days tetracycline schedule with the biopsy 2 days following the second label. Vertical trephine bone biopsies were taken from the anterior iliac crest and fixed in 1:1 10\% buffered formalin phosphate and $0.5 \%$ sucrose solution for $2 h^{4}{ }^{4}$ They were then dehydrated in propanol and embedded in glycol methacrylate. ${ }^{5}$ The entire procedure was carried out at $4^{\circ} \mathrm{C}$. Blocks prepared prior to 1981 in either Epon or methyl methacrylate were also used to examine aluminium staining. Sections $(\geqslant 2 \mu \mathrm{m})$ were cut using a Porter-Blum MT2 microtome and glass knives. Mean total section area was $2.0 \pm 0.1 \mathrm{~cm}^{2}(\mathrm{n}-$ $=10$ ). Sections were stained for acid phosphatase ${ }^{6} \mathrm{O}$ and toluidine blue, iron and Giemsa using standard\$ techniques. Five per cent desferoxamine in either $\frac{3}{\sigma}$ saline or phosphate buffer was applied directly toळ the sections for $30 \mathrm{~min}$ at $37^{\circ} \mathrm{C}$ as was $1 \%$ acetic acid $\vec{\circ}$ or $10 \%$ EDTA, the latter at room temperature. Aluminium staining ${ }^{2}$ was done on $8 \mu \mathrm{m}$ thic $\mathrm{k}$ sections without counterstain for recognition ofo bone or marrow aluminium. Fluorescence was looked for using unstained section $2-8 \mu \mathrm{m}$ in thick-o ness. Reactivity for non-specific esterase was examined in embedded tissue within two weeks $\stackrel{0}{2}$

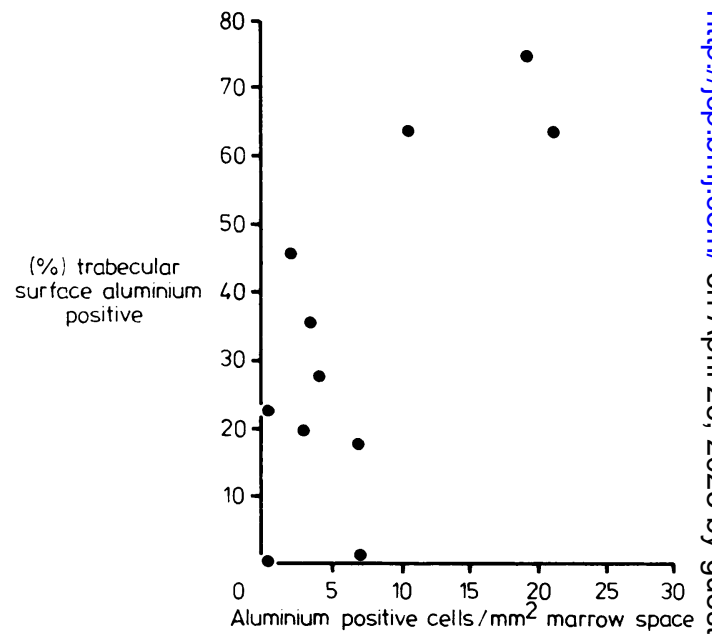

Fig. 1 Lack of close relation between bone and marrow staining for aluminium. 

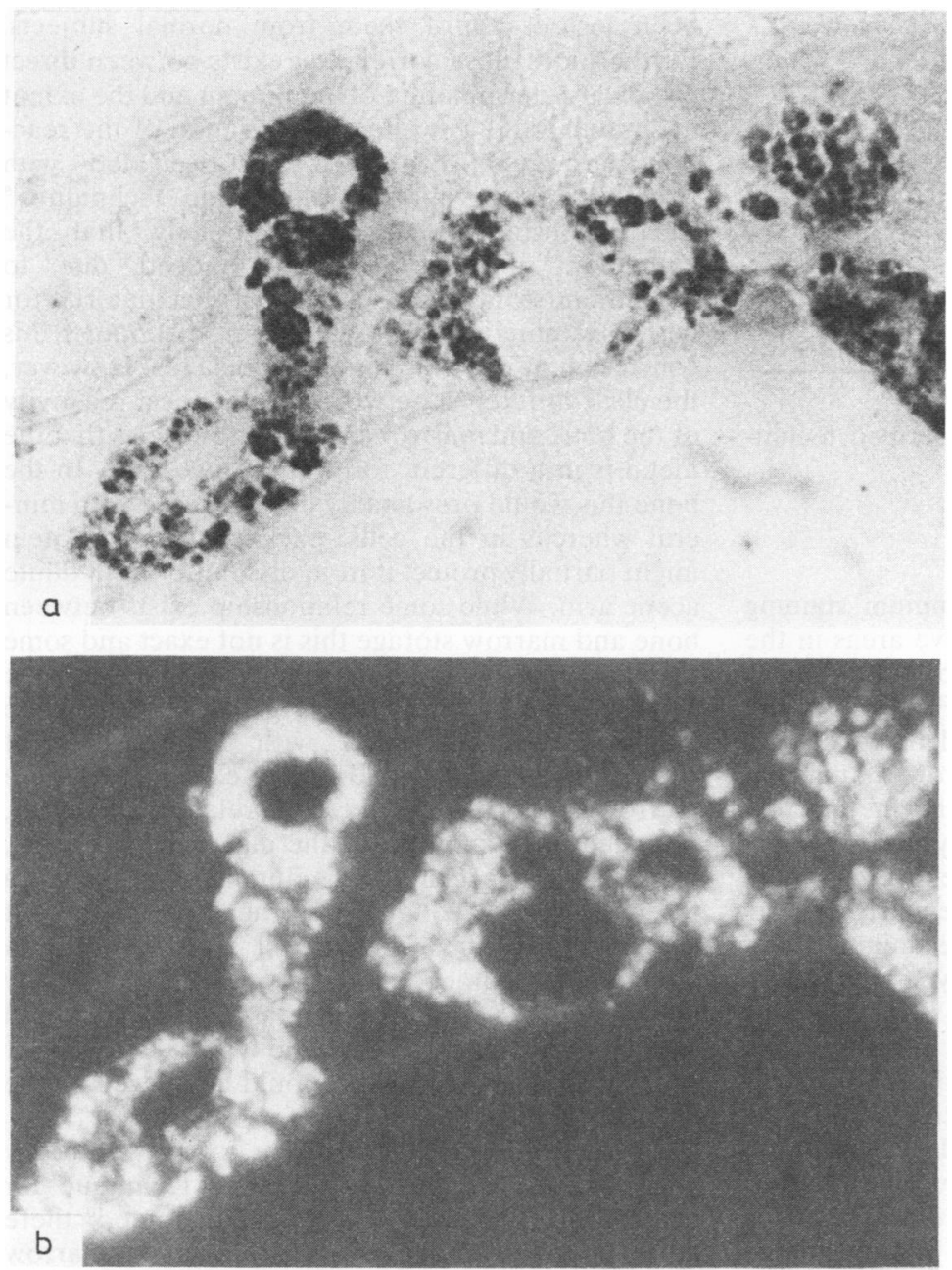

Fig. 2 Original magnification $\times 1000$, 2 um sections.

(a) Aluminium stain showing positive areas in the marrow. The granular structures are stained bright red; (b) fuorescent picture of the same section. The patient had received tetracycline according to the protocol; (c) toluidine blue stain of the next section showing the coarse granules.

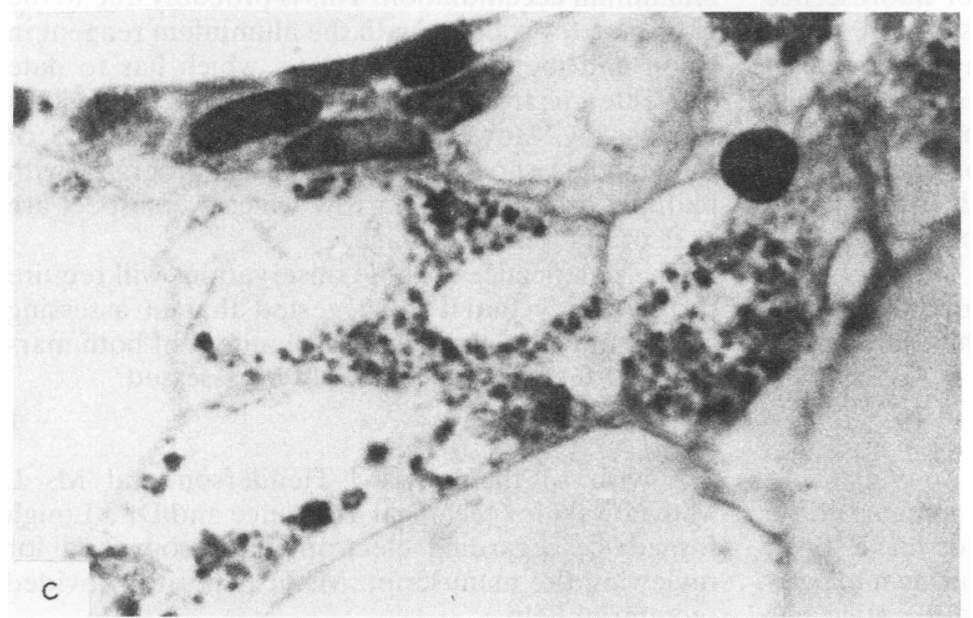


Effect of section pretreatment on subsequent aluminium staining in a representative patient

\begin{tabular}{lll}
\hline Pretreatment & \multicolumn{2}{c}{ Aluminium staining } \\
\cline { 2 - 3 } & Marrow & Bone \\
\hline None & +++ & ++++ \\
EDTA & - & - \\
1\% acetic acid & ++ & - \\
$\begin{array}{l}\text { Desferoxamine } \\
\text { In phosphate buffer pH 7.2 }\end{array}$ & - & +++ \\
In saline pH 4.9 & - & ++ \\
Saline or buffer alone & +++ & ++++ \\
\hline
\end{tabular}

of procurement using previously described techniques. $^{57}$

\section{Results}

The majority of biopsies with aluminium staining areas in the bone also showed positive areas in the marrow (Fig. 1). These appeared as bright red areas and corresponded to marrow cells (Fig. 2a). Of the 28 patients, in 18 the bone stained positively for aluminium and 11 of these had marrow staining. In one patient the bone was negative and the marrow positive and one patient had strongly positive marrow and only a small amount in bone. In two others the bone was strongly positive and the marrow negative. With Giemsa and toluidine blue staining these cells appeared to be macrophage in type with coarse granules in the cytoplasm (Fig. 2c). Iron stain was negative. The effect of pretreatment of the sections with different reagents is shown in the Table for a representative patient who showed strongly positive staining in both bone and marrow. Desferoxamine removed all staining from the marrow cells but had much less effect on bone, acetic acid did the reverse and EDTA abolished the staining in both areas. Examination of unstained sections for fluorescence showed that aluminium positive cells gave a strong yellow fluorescence from granules in the cytoplasm (Fig. 2b). However in patients who had not been labelled with tetracycline prior to biopsy, fluorescence was absent, although a non-specific less intense fluorescence was sporadically seen in the marrow unrelated to aluminium reactivity. Positive aluminium staining was never found in osteoblasts. Osteoclasts, whether identified by routine staining or by the more sensitive acid phosphatase reaction were also negative.

\section{Discussion}

The staining reaction for aluminium appears to be quite specific and no false positives have been reported. This is in agreement with our own experience in more than 70 biopsies where the stain has been tested, 13 of them from normal subjects. Furthermore close correlation exists between direct $\underline{\underline{\sigma}}$ chemical determination of aluminium and the extent of staining ${ }^{2}$ and as well the localisation of the reaction has been confirmed as associated with aluminium using more sophisticated techniques. ${ }^{8}$ 음 These considerations make it likely that the $\frac{\overline{\bar{\rho}}}{\overline{0}}$ aluminium positive cells are indeed due to $\mathbb{Q}$ aluminium storage. The affinity of this material for desferoxamine in the absence of iron supports this conclusion as does the binding to EDTA. However, $\stackrel{\circ}{.}$ the clear difference between the chemical reactivity $\overrightarrow{\vec{\omega}}$ of the bone and marrow aluminium suggests that the $\stackrel{\sigma}{\omega}$ metal is in a different form in the two sites. In the bone this would presumably be associated with min- $\dot{\omega}$ eral whereas in the cells, packaging with protein might partially protect it from dissolution with dilute $\overrightarrow{.}$ acetic acid. While some relationship exists between $\vec{N}$ bone and marrow storage this is not exact and some $\underset{\infty}{\infty}$ patients have positive bone staining and none in the marrow and the reverse (Fig. 1). If the bone aluminium is associated with the process of $z$ calcification then it would not be expected that the marrow storage would be closely related. The latter, if indeed involving cells of the macrophage system, $\frac{\mathbb{Q}}{\mathbb{D}}$ would then be accumulating aluminium as part of a foreign body (? colloidal) storage similar to tha described for silicon.9 The cell type involved aluminium storage has not been positiver identified. The negative esterase reaction would suggest these are not monocytic type cells, however their staining characteristics could be altered by the aluminium accumulation.

To our knowledge, except for a brief report by $\stackrel{2}{\Rightarrow}$ Ackrill $e t$ al using an unpublished technique for $\frac{9}{3}$ characterising aluminium with Solachrome, ${ }^{10}$ there have been no previous descriptions of marrow aluminium accumulation. This is probably due to the poor marrow staining with the aluminium reagent in ? Epon and methyl methacrylate, which has to date prevented us from examining these cells by electron microscopy. However, Maloney et al in Fig. 5 of $\delta$ their paper ${ }^{2}$ show a fluorescence photograph with $₹$ multiple areas in the marrow which we suspect are $\frac{9}{5}$ sites of aluminium storage.

The significance of these observations will require further study but it is suggested that in assessing $\bar{N}$ aluminium storage in bone the content of both mar- row and the bone itself should be assessed.

We wish to thank Ms J Henderson and Ms Malynowski for technical assistance and Dr J Lough for advice regarding electron microscopy and for $\stackrel{\text { ? }}{?}$ reviewing the manuscript. Ms $\mathrm{H}$ Esposito provided 7 secretarial help. 
This study was supported by a grant from the Kidney Foundation of Canada.

\section{References}

'Buchanan MRC, Ihle BV, Dunn CM. Haemodialysis related osteomalacia: a staining method to demonstrate aluminium. $J$ Clin Pathol 1981;34:1352-4.

${ }^{2}$ Maloney NA, Ott SM, Alfrey AC, Miller N, Coburn JW, Sherrard DJ. Histological quantitation of aluminium in iliac bone from patients with renal failure. J Lab Clin Med 1982;99(2):206-16.

${ }^{3}$ Smith PS, McClure J. Localisation of aluminium by histochemical and electron probe $\mathrm{X}$-ray microanalytical techniques in bone tissue of cases of renal osteodystrophy. J Clin Pathol 1982;35:1283-93.

${ }^{4}$ Fallon MD, Teitlebaum SL. A simple procedure for the rapid histologic diagnosis of metabolic bone disease. Calcif Tissue Internat 1981;33:281-3.

${ }^{5}$ Westen H, Muck KF, Post L. Enzyme histochemistry on bone marrow sections after embedding in methacrylate at low temperatures. Histochemistry 1981;70:95-105.

- Evans RA, Dunstan CR, Baylink DJ. Histochemical identification of osteoclasts in undecalcified sections of human bone. Mineral and Electrolyte Metabolism 1979;2:179-85.

'Yam LT, Li CY, Crosby WH. Cytochemical identification of monocytes and granulocytes. Am J Clin Pathol 1971;55:28390

${ }^{8}$ Cournot-Witmer G, Zingraff J, Plachot JJ, et al. Aluminium localisation in bone from hemodialysed patients. Relationship to matrix mineralisation. Kidney Int 1981;20:375-85.

${ }^{9}$ Leong ASY, Disney APS, Goue DW. Refractile particles in liver of haemodialysis patients. Lancet $1981 ; \mathrm{i}: 889$.

${ }^{10}$ Ackrill P, Day JP, Gorstang FM, et al. Treatment of fracturing renal osteodystrophy by desferoxamine. Proc EDTA 1982; 19:203-7.

Requests for reprints to: Dr M Kaye, Division of Nephrology, The Montreal General Hospital, 1650 Cedar Avenue, Montreal, Quebec, H3G 1A4, Canada. 